\title{
Principios éticos y legales en la práctica pericial psiquiátrica.
}

Ethical and legal principles at the psychiatric expert practice.

\section{J. González ${ }^{1,2}$, A. Rapún ${ }^{1,3}$, R. Altisent ${ }^{4}$ y J. Irigoyen ${ }^{1}$}

\section{RESUMEN}

Se plantea un análisis ético de la práctica pericial psiquiátrica, realizando una reflexión crítica a partir de las referencias clásicas de la bioética y la deontología, aplicadas a la relación clínica convencional.

Se concluye con una selección de los principios éticos que, adaptados a la especificidad de la psiquiatría forense, podrían servir de referencia para la excelencia en la práctica de esta rama de la medicina legal.

Palabras clave: Bioética, Deontología, Ética Médica, Psiquiatría Forense.

Cuad Med Forense 2005; 11(42):275-285

\section{ABSTRACT}

An ethical analysis of psychiatric expert practice has been raised, carrying a critical thought out from classic references of bioethics and deontology, applied to conventional clinical relation.

Concluding with a selection of the ethical principles which, adapted to the specifity of forensic psychiatry, could make use of reference superbly to the practice of this subspeciality of legal medicine.

Key words: Bioethic, Deontology, Forensic Psychiatry, Medical Ethic.

Correspondencia: Jorge González Fernández. Instituto de Medicina Legal de La Rioja. C/ Hermanos Hircio nº 5. 26005 - LOGROÑO. E-mail: JorgeGonzalez@saludalia.com.

1 Médico Forense, Especialista en Medicina Legal y Forense, Instituto de Medicina Legal de La Rioja.

2 Postgrado en Psiquiatría (Universidad de León).

3 Master en Valoración del Daño Corporal (Universidad del País Vasco).

4 Profesor de Bioética. Instituto de Bioética y Ciencias de la Salud. Universidad de Zaragoza. 


\section{"Cuando los tribunales o autoridades apelan a nuestro ministerio para que emitamos una opinión acerca de los hechos que someten a nuestro juicio, no es solamente ciencia lo que piden, sino también moralidad".} Pedro Mata.[I]

\section{INTRODUCCIÓN:}

La habitual relación médico-paciente que rige por principio toda actuación terapéutica, queda modificada en la práctica pericial psiquiátrica por el diferente objetivo de la evaluación médica.

Si en una relación clínica el enfermo traslada al médico sus síntomas, y este observa los signos con el objetivo de concluir un diagnóstico que permita instaurar un tratamiento por el que la salud del paciente se restituya, en la relación pericial que se establece entre el sujeto del informe y el perito evaluador se trata de valorar esos síntomas referidos y esos signos observados con el objetivo de determinar si el individuo conserva sus capacidades de entender la realidad que le rodea y de obrar conforme a dicha comprensión. Y todo ello tanto en el ámbito jurisdiccional penal, en el que estas capacidades constituyen el sustrato psicobiológico de la imputabilidad, como en el civil, en el que se determinará si conserva sus capacidades para el gobierno de su persona y sus bienes, incluyéndose en estos casos su posible incompetencia en aquellas situaciones en las que una patología haga necesario su internamiento en centro psiquiátrico.

\section{APLICACIÓN DE LA BIOÉTICA A LA PSIQUIATRÍA FORENSE:}

Al objeto de centrar la cuestión bioética en esta rama tan concreta de la medicina, debe en primer lugar comprenderse el concepto de psiquiatría legal, que puede definirse como el conjunto de conocimientos psicológicos y psicopatológicos precisos para la resolución de los problemas que plantea el Derecho, tanto en lo que se refiere al desarrollo y perfeccionamiento de este como en cuanto a la aplicación práctica y justa de las Leyes.

Desde una perspectiva práctica, la Psiquiatría Forense constituye una ayuda técnica que se presta al legista en un doble campo principalmente: en el plano criminal (Código Penal, Ley de Enjuiciamiento Criminal) y en el civil (Código Civil y Ley de Enjuiciamiento Civil) y a un nivel más secundario en diferentes ámbitos (Código canónico, Legislación laboral...).

La American Academy of Psichiatry and the Law (AAPL) define la Psiquiatría Forense como "Una subespecialidad de la psiquiatría en la que un peritaje científico y clínico se aplica a aspectos legales en conceptos legales que abarcan materias civiles, penales, penitenciarias o legislativas" [2].

Por otra parte, en la Encyclopedia of Bioethics editada en 1.978, se definía la bioética como un área de investigación que, valiéndose de una metodología interdisciplinaria, tiene por objeto "el estudio sistemático de la conducta humana en el campo de las ciencias de la vida y de la salud, analizando esta conducta a la luz de los valores y principios morales" [3].

Con lo anterior, puede decirse que la aplicación de los principios de la Bioética al campo de la Psiquiatría Forense tendría por objeto la evaluación de la conducta profesional del médico-perito en cuanto a la utilización de sus conocimientos psicológicos y psicopatológicos para la resolución de las cuestiones que plantea el Derecho.

\section{PRINCIPIOS ÉTICOS EN PSIQUIATRÍA FORENSE:}

La aplicación de unos principios éticos a la Psiquiatría Forense deberá sustentarse en la adaptación de las obligaciones morales del perito a los derechos del paciente, y en la singular relación que surge entre ambos, que debe adecuarse a las exigencias que para este tipo de 
actuaciones médicas plantea el Código de Ética y Deontología, guardando todo ello un perfecto equilibrio con el fin último de que el acto médico a realizar, en este caso el acto pericial, se enfoque hacia el mejor asesoramiento a jueces y tribunales acerca de los problemas psicopatológicos que pudiera presentar el informado.

Para ello, conviene revisar los diferentes conceptos éticos que integran esta particular relación enfermo-informado / médico-perito.

\section{I.- Principios básicos de la bioética:}

El ejercicio de la medicina, en cualquiera de sus vertientes debe realizarse atendiendo a unos principios éticos de actuación, que fueron ya definidos en 1.979 por Beauchamp y Childress [4], y más tarde categorizados por Diego Gracia en función de su universalidad para cualquiera de los ámbitos de la relación en sociedad o de su particularidad derivada de la relación médico-paciente [5].

Entre los primeros, que afectan de modo universal a cualquier relación en sociedad, dos son los principios que rigen cualquier actuación médica:

- El principio de no-maleficencia por el que el médico, al igual que cualquier persona, debe evitar en sus conductas la realización de un mal a sus semejantes o a lo que le rodea, bajo el principio del "Primum non nocere".

- El principio de justicia, entendido como concepto de equidad, por el cual todos los miembros de la sociedad deben ser considerados iguales, sin que ello permita discriminaciones en el ejercicio profesional en razón de edad, sexo, raza, religión o cualquier otra circunstancia. Implica una consideración imparcial en la distribución de recursos.

Además de los dos principios antes mencionados, la relación médico-paciente debe basarse, por las propias características de la labor asistencial, en otros dos, que son:

- El principio de beneficencia: que se basa en la pretensión de que la actuación médica resulte beneficiosa para el paciente procurándole un bien objetivo, de tal modo que en la actuación médica se potencien los beneficios, y se minimicen en lo posible los riesgos.

- El principio de autonomía, entendiéndose por tal la capacidad del paciente para participar de un modo libre y consciente en las posibilidades terapéuticas que conduzcan a una restitución o mejora de su salud, a través del conocimiento lo más amplio posible de su patología y de las opciones que respecto a su tratamiento pueden plantearse [6].

\section{Normas derivadas de la relación médico-paciente:}

Toda relación médico-paciente se rige además de por los pilares bioéticos básicos expuestos en el apartado anterior, por una serie de normas que desarrollan a las anteriores:

- Confidencialidad: El médico tiene la obligación ética de guardar reserva de cuanta información sea proporcionada por el propio paciente, o se obtenga a través de las exploraciones y pruebas complementarias que se realicen, debiendo quedar plenamente garantizada la privacidad de cualquier dato que le pudiera ser proporcionado, con las salvedades dispuestas por la normativa legal. Este principio ético ya quedaba reflejado en el compromiso 7 del juramento hipocrático -"Todo lo que viere y oyere en el ejercicio de mi profesión, y todo lo que supiere acerca de la vida de alguien, si es cosa que no deba ser divulgada, lo callaré y lo guardaré con secreto inviolable"-, y se encuentra recogido en el Código de Ética y Deontología español, que contempla la confidencialidad como un deber del médico y un derecho del paciente [7]. 
- Fidelidad: Directamente derivada de la relación de lealtad que recíprocamente se establece entre el paciente y su médico, constituyendo lo que ha venido a denominarse "alianza terapéutica", que resulta imprescindible en el ámbito de la psiquiatría para obtener una mejora en la salud a través de la confianza y el respeto mutuos que permite la adopción autónoma del tratamiento adecuado.

- Consentimiento informado: Necesario para que el paciente ejerza su derecho de autonomía a través de la comprensión lo más exacta posible de su proceso y de las alternativas terapéuticas existentes [8].

\section{III.- Deberes y obligaciones del perito psiquiatra:}

La particular función del perito psiquiatra no tiene como objetivo emitir un diagnóstico para, a través de un tratamiento, conseguir la restitución de la salud del paciente, sino la de proporcionar al juzgador una información que le permita aplicar justicia.

De ahí que, a los principios bioéticos que presidirán toda actuación médica, deben añadirse una serie de normas que acompañarán a los anteriores para garantizar su cumplimiento. Las más importantes son: objetividad, prudencia, reflexión, juicio, imparcialidad, veracidad $[9,10]$ y honestidad. Veámoslas:

I. Objetividad: Norma fundamental para la interpretación de las pruebas y resultados obtenidos sin atender a posibles prejuicios o condicionantes, que pueden derivar de los propios hechos o de las circunstancias particulares de la persona a explorar.

2. Prudencia en la elaboración de dictámenes, con capacidad para no asumir verdades absolutas a través del conocimiento de las propias limitaciones, atendiendo a lo expuesto por Brouardel hace ya un siglo "la calidad mayor que debe tener el perito no es la extensión de sus conocimientos, sino la noción exacta de lo que sabe y de lo que ignora" [I I].

3. Reflexión y juicio: al objeto de racionalizar los hallazgos razonando de manera lógica sobre ellos, simplificando los problemas que pueden plantearse y jerarquizando lo principal sobre lo accesorio, hasta alcanzar las conclusiones válidas.

4. Imparcialidad: el psiquiatra perito debe expresar sus opiniones científicas prescindiendo de cualquier implicación afectiva sobre los hechos valorados, o sobre cualquiera de las partes contrapuestas en el procedimiento, realizando su valoración con independencia de las consecuencias jurídicas y sociales que puedan derivar de ella. Este principio de imparcialidad se resume en que el psiquiatra forense debe ser siempre y únicamente perito de la verdad.

5. Veracidad: El perito debe emplear un método que permita contrastar las conclusiones científicamente obtenidas, debiendo constituirse este principio de veracidad como uno de los soportes primordiales que guíe la prueba pericial forense.

6. Honestidad: Por la cual el perito permanecerá ajeno a cualquier interés espurio en las evaluaciones que se le encomienden.

Además de lo anterior, el psiquiatra forense, de manera evidente, deberá procurarse una correcta formación en su rama del saber, a la que añadirá suficientes conocimientos jurídicos que le permitan transmitir de manera clara y comprensible sus conclusiones a los profesionales del derecho destinatarios. 


\section{IV.- Características especiales de la relación sujeto evaluado - perito:}

La relación sujeto-perito no puede ser tenida por una alianza paciente-terapeuta como tal, en razón de las circunstancias especiales que confluyen en ella.

- La primera cuestión destacable es el objetivo último de la relación médico-paciente, que para el caso de tratarse de una evaluación pericial no va a ser el beneficio para la salud del informado, sino las posibles consecuencias legales que pueden derivar de su patología, bien referentes a su imputabilidad -cuando se trate de materia penal- o a su competencia en la administración de su persona o bienes -en cuestiones civiles-.

- Otra particularidad viene determinada por la diferente persona que solicita la intervención del psiquiatra forense, y que si en una relación clínica y terapéutica es habitualmente el propio enfermo o un allegado, en el caso de la valoración pericial es el juez o cualquiera de las partes implicadas. Esta circunstancia puede llegar a condicionar de manera importante esa alianza o empatía que de modo indudable facilitaría que el explorado proporcionase los datos que permitan dar cumplimiento al objeto del reconocimiento. Esta misma circunstancia viene a condicionar la debida confidencialidad de la información proporcionada, por cuanto será empleada para la elaboración del informe solicitado. Por ello, en cualquier caso, y siguiendo lo dispuesto en el Código de Ética y Deontología Médica [7], el sujeto evaluado deberá ser informado exhaustivamente del objeto de la exploración forense, así como de la parte que lo solicita, a fin de que pueda decidir de modo libre acerca de su disposición a ser reconocido, según sus intereses.

- Respecto al lugar en el que se establece la relación médico-enfermo -juzgados, dependencias policiales, cárcel, psiquiátrico...- debe ser igualmente tenido en cuenta, por cuanto generalmente dista de ser el idóneo para el establecimiento de una adecuada empatía que favorezca la relación de confianza entre el médico y el informado, con la consecuente dificultad para que el reconocimiento pueda ser abordado en condiciones que garanticen el pleno éxito de la evaluación pericial.

- Una última diferencia destacable reside en el objeto documental sobre el que recae la información: En el caso de la evaluación pericial el psiquiatra forense no plasma la información en una historia clínica, sino en un dictamen cuyo contenido viene contemplado en el artículo 478 de la Ley de Enjuiciamiento Criminal: " I Descripción de la persona o cosa que sea objeto del mismo en el estado o del modo en que se halle. $2^{\circ}$ Relación detallada de todas las operaciones practicadas por los peritos. $3^{\circ}$ Las conclusiones que en vista de tales datos formulen los peritos, conforme a los principios y reglas de su arte" [12]. La propia característica que como documento público tiene el informe pericial, hace que la información obtenida pierda cualquier posibilidad de privacidad, al quedar a disposición en primer lugar de los funcionarios judiciales, y más tarde de los abogados de ambas partes, de los fiscales, jueces, procuradores, y otros peritos contrapuestos. Este documento, por ello, no preserva en su contenido el principio de confidencialidad, al hacerse público, de tal modo que como ya se aclaró anteriormente, deberá informarse exhaustivamente al sujeto de la pericia sobre los fines del reconocimiento.

\section{RECOMENDACIONES ÉTICAS EN PSIQUIATRÍA FORENSE:}

Todo lo expuesto hasta el momento provoca, sin duda, una colisión entre los principios éticos que deben regir la clásica y convencional relación médico-paciente, con las particularidades de las obligaciones del perito y las circunstancias especiales que aparecen en la relación sujeto-evaluador. 
Para realizar este análisis, conviene diferenciar la intervención en materias penales, en las que se pretende una evaluación de las bases psicobiológicas de la imputabilidad, de las valoraciones en el ámbito civil, en el que sí existe un interés en la protección del enfermo en la medida en la que el conocimiento de su posible incompetencia permite establecer medidas orientadas a la salvaguarda de su integridad patrimonial o a la autorización de un tratamiento enfocado a la restitución de su salud.

\section{A.- ACTUACIONES EN EL ÁMBITO PENAL:}

El objetivo principal de la pericia en este campo es la determinación del estado mental en el momento de la comisión de los hechos. También, aunque en menor proporción, puede tratarse de la posibilidad de aplicación de medidas de seguridad, o evaluaciones sobre la capacidad para prestar testimonio, la competencia para ser juzgado o la existencia de posibles secuelas psíquicas.

En esta jurisdicción, la correcta evaluación pericial no exige alcanzar un diagnóstico concreto, sino evaluar en que medida están afectadas las bases psicobiológicas de la imputabilidad -la inteligencia y la voluntad- esto es, la capacidad de comprender lo ilícito y de obrar conforme a tal entendimiento. Esta afirmación puede realizarse de manera categórica recordando para ello que la función del psiquiatra forense, y por tanto la finalidad de su informe pericial, es evaluar los posibles determinantes psicopatológicos de las conductas del procesado, es decir, su imputabilidad -de ninguna manera cuestiones de derecho como la responsabilidad- y ello porque no puede atribuirse un significado a una conducta humana sin que se den determinadas condiciones intelectivas y volitivas que le capaciten para una cabal comprensión.

Una vez definido el objeto de la actuación psiquiátrica pericial, se procederá a describir las normas éticas que deben regirla, atendiendo a los soportes básicos descritos en los apartados anteriores:

- En primer lugar, podemos observar una posible colisión entre el principio básico de nomaleficencia y la evaluación psiquiátrica forense. La obligación del médico como tal exige, como hemos visto al hablar de los pilares de la bioética en medicina, evitar en sus conductas la realización de un mal a sus semejantes o a lo que le rodea, bajo el principio del "Primum non nocere" que podría interpretarse como "la máxima prioridad, no dañar".

Puede darse el caso de que tras la evaluación pericial y la elaboración de unas conclusiones se derive un perjuicio al entender que el individuo cometió el ilícito penal en condiciones de conservación suficiente de sus capacidades de entender y de obrar, de lo que derivaría la posible imposición de una sanción que evidentemente resulta perjudicial para sus intereses, ocasionándole en sentido estricto un daño.

A este respecto, debe recordarse que si bien el informado asume en esta relación el rol de paciente, el psiquiatra forense se debe éticamente a esa instancia superior para la que realiza su labor pericial y que ha sido reseñada someramente en el apartado de obligaciones del perito psiquiatra cuando se decía que el forense debe ser siempre y únicamente perito de la verdad, engarzándose esta afirmación con el principio de justicia que debe ser interpretado en su amplia extensión, más allá del mero concepto de equidad en la distribución de los recursos.

El psiquiatra forense por tanto, deberá supeditar la aplicación estricta de ese principio de no-maleficencia, al criterio de justicia en su amplio sentido, valiéndose para ello de la verdad y de la honestidad.

- El segundo punto de colisión aparece al analizar el principio de autonomía, por el cual, el paciente tomará de modo libre sus decisiones conforme a la exhaustiva información que debe ser proporcionada por el médico. 
Siguiendo los criterios de la American Academy of Psychiatry "Siempre que sea posible se debe obtener el consentimiento informado del sujeto sometido a un examen forense. Cuando no sea preceptivo, hay que mencionar al examinando la naturaleza de la evaluación. Si el sujeto no es competente para otorgarlo, se ha de obtener un consentimiento por sustitución según las leyes de cada jurisdicción" [2]. A este respecto, la ausencia de un consentimiento expreso libremente elegido puede conllevar una intrusión ilegítima en áreas de la intimidad que podrían cuestionar el fundamento y el sentido del informe, haciéndolo éticamente inaceptable.

De tales premisas pueden derivarse dos situaciones diferenciadas, siendo la primera de ellas que el informado no sea mentalmente competente para comprender el significado o las consecuencias de la exploración a la que va a ser sometido. Tal planteamiento no deja de ser trascendente por cuanto ante un paciente psiquiátrico cabe cuando menos cuestionarse si es capaz de recibir información y elaborarla, y si puede hablarse de voluntad y entendimiento no viciados que le hagan competente para prestar un consentimiento válido. Si una circunstancia de privación de su competencia se diese, y el inculpado no fuese capaz de discernir el alcance de la exploración, podría proporcionar datos al psiquiatra forense que le resultasen perjudiciales, lo que plantea un compromiso ético evidente en la pericial resultante [13]. No obstante lo anterior, en ocasiones la mera observación del sujeto evaluado por un psiquiatra forense experto puede aportar datos que permitan una suficiente valoración del compromiso psicopatológico de las diferentes esferas de su psiquismo, en relación con los hechos que se le imputan.

La segunda situación posible, en relación con la autonomía del informado respecto a su libre elección a ser reconocido, pasa por su negativa a someterse a la exploración psiquiátrica. El individuo puede de modo libre y consciente negarse a prestar el consentimiento para someterse a una evaluación forense, asistiéndole no sólo el derecho a ello, sino la obligación ética del perito, contemplada en el Código de Ética y Deontología -en su artículo 41.2- de renunciar a la práctica del reconocimiento [7].

- Continuando con el análisis de las normas éticas que deben regir la actuación pericial psiquiátrica, llegamos al deber de confidencialidad, observando como para ella surgen nuevamente tensiones entre la obligación como psiquiatra de guardar reserva de la información proporcionada por el paciente u obtenida por otros medios, y la obligación como perito de plasmar en un documento -informe- las conclusiones que se deriven de los datos obtenidos, documento que quedará a pública disposición de las partes enfrentadas en el proceso.

A este respecto, la AAPL señala "El respeto del derecho de un individuo a la privacidad y al mantenimiento de la confidencialidad debe ser una preocupación fundamental para un psiquiatra que realiza evaluaciones forenses. El psiquiatra debe proteger la confidencialidad lo más posible dentro del contexto legal. Está obligado a prestar una atención especial a cualquier limitación a los preceptos habituales de la confidencialidad médica. Una evaluación con fines forenses debe comenzar anunciando a la persona examinada cualquier restricción de la confidencialidad. La información o los informes derivados de la evaluación forense deben someterse a las mismas reglas de confidencialidad que la propia evaluación y cualquier divulgación debe quedar restringida en el mismo sentido" [2]. 
A tales efectos, las normas éticas que deben regir al psiquiatra forense exigen una explicación clara al sujeto evaluado de que la información obtenida que se considere relevante para la pericial quedará reflejada documentalmente, debiendo garantizar el respeto a la confidencialidad de toda aquella información que no sea de interés estricto para los propósitos legales de la evaluación, de tal modo que tan solo se incluirán las observaciones que resulten imprescindibles para sustentar las conclusiones finales.

Una nueva cuestión surge en este punto, como es el tratamiento que debe darse a aquellos datos complementarios obtenidos del material clínico (historia, informes, referencias de familiares...) aportados al objeto de contrastar las informaciones proporcionadas por el sujeto-paciente, y que plantean el dilema ético sobre la revelación de datos que puedan resultar de interés para el procedimiento penal [13]. A este respecto, debe entenderse que la confidencialidad documental queda relevada bien mediante el consentimiento explícito del informado si es este quien la aporta, o bien a través de la posible publicidad que adquiere el documento de haber sido incluido en los autos judiciales por alguna de las partes implicadas.

- Otra norma ética, con la que el perito psiquiatra debe ser especialmente cuidadoso es la objetividad, que puede verse en ocasiones afectada por la propia condición de médico, que determina, al menos teóricamente, una tendencia natural a ayudar al paciente. En este caso, no debe guiar esa natural beneficencia al objeto de la pericia, resultando obligado mantener lo que Bonnet denomina acertadamente "impermeabilidad sugestiva" [14] que permita conservar la objetividad exigida como garantía de imparcialidad de la prueba pericial.

- Por último, la norma ética de honestidad, que obliga a mantener la independencia en la actuación científica respecto de la parte para la que se actúa, y que para la AAPL se expresa del siguiente modo: "Los psiquiatras forenses actúan como peritos en el seno de los procesos legales. Aunque puedan ser contratados por una de las partes en un litigio de carácter civil o por la acusación o la defensa en un asunto penal, están sujetos al principio de honestidad y persiguen la objetividad. La evaluación clínica que realicen y la aplicación de los datos obtenidos en la misma a los criterios legales deben realizarse en el espíritu del citado principio y de los esfuerzos por alcanzar la objetividad. Esto mismo debe quedar reflejado en el dictamen" [2]. De tal principio se desprende la obligación del perito de actuar imparcialmente con independencia absoluta de cual sea la parte contratante o que solicita su pericia. La honestidad obliga al perito psiquiatra a emitir el dictamen al margen de dichas consideraciones, y de elevar a conclusiones sus hallazgos aún cuando los mismos entren en colisión con los intereses de quien le propuso, correspondiendo a este último, de estimarlo procedente, determinar las estrategias sobre el litigio que considere más adecuadas para sus intereses.

Respecto a esta norma de honestidad, que debe regir la actuación pericial psiquiátrica incluso más allá de una idónea actitud ética en la vida ordinaria, deben despreciarse en el sentido literal del término los honorarios condicionados, estos son los ligados al resultado de la causa, por cuanto pueden condicionar la pericial realizada relegando los principios insobornables de honestidad y objetividad, justificando el aforismo de Gutheil respecto a que "el perito íntegro vende su tiempo, el mercenario vende su testimonio" [15].

En este punto, cabe citar a Jiménez Cubero cuando expresa que "Un informe psi- 
quiátrico es una obra espiritual, es una producción del espíritu que presupone ciencia" [16] por lo que pruebas periciales elaboradas por distintos peritos forenses no tienen necesariamente porqué ser coincidentes en sus conclusiones, sin que por ello quepa presumir una falta de honestidad o de objetividad, resultando tan sólo la constatación de que las cuestiones psiquiátrico forenses, por su complejidad, no revisten una certeza absoluta sino tan sólo una certeza médica razonable [13].

\section{B.- ACTUACIONES EN EL ÁMBITO CIVIL:}

Las actuaciones psiquiátrico-forenses en esta materia tienen lugar en el ámbito de la salvaguarda de los intereses del enfermo, tanto cuando se trata de intervenciones en procedimientos de incapacitación, como en aquellos cuadros en los que la descompensación de una posible patología haga necesario su internamiento en centro psiquiátrico cerrado.

Estas dos situaciones van a plantear diferentes cuestiones bioéticas en la relación peritoenfermo:

\section{I.- Evaluación de competencia:}

En esta, deben regir los mismos principios éticos hasta ahora revisados, pudiendo observarse una colisión en materia de autonomía para consentir ser sometido a un reconocimiento, causada por la propia incompetencia inherente al procedimiento de incapacidad que lo motiva. Resulta en todo caso obvio que la autonomía del paciente puede hallarse comprometida, puesto que el origen de la evaluación forense radica precisamente en el cuestionamiento de su competencia, lo que de por sí hace igualmente cuestionable su capacidad para comprender el alcance del reconocimiento y su libre voluntad para someterse al mismo, afectando a su capacidad para otorgar un consentimiento informado respecto a las exploraciones a realizar y sus fines.

A estos efectos debe primar el principio bioético de beneficencia, esto es, el de efectuar el reconocimiento para la mejor protección de persona y patrimonio del informado, todo ello siempre bajo la premisa de tratarse de una evaluación que será sometida a un control judicial que garantice todos los derechos del enfermo.

Respecto al principio de honestidad, el perito psiquiatra será especialmente cuidadoso en aquellas situaciones en las que intervenga a propuesta de parte en evaluaciones psiquiátricas relacionadas con testamentarías, en las que ocasionalmente se pretenden modificaciones alegando o valiéndose de patologías incapacitantes en el momento de prestar las nuevas disposiciones. A este respecto, y tal como se expuso, el psiquiatra forense deberá dejarse guiar por la honestidad sin atender a los intereses concretos de la parte que le propone como perito.

\section{2.- Internamiento psiquiátrico no consentido:}

El principal dilema ético en relación con los internamientos forzosos, radica en el posible cuestionamiento de la capacidad del paciente para otorgar consentimiento respecto a su necesidad de recibir tratamiento en centro psiquiátrico cerrado, lo que por otra parte afecta a uno de sus derechos fundamentales contemplado en el artículo 17. I de la Constitución de I.978 [17], el derecho a la libertad.

Así, el principio de autonomía del paciente, por el cual, de ser este competente, debe participar de manera libre y consciente en cuantas decisiones afecten a su tratamiento tras recibir la información necesaria, choca frontalmente con la posible alteración que la propia patología determina en sus capacidades de entender y de obrar. 
A este respecto debe considerarse que, aún existiendo una patología que comprometa su comprensión y su voluntariedad, el criterio a seguir será el de la flexibilidad, y dado que un internamiento no supone necesariamente estar mermado en las funciones psíquicas superiores que sustentan el concepto social y jurídico de la capacidad para la libre autodeterminación, cabe aceptar el denominado consentimiento moderado frente a la rigurosidad del consentimiento estricto [ 18$]$. Con ello puede evitarse la burocratización antiterapéutica que supondría el que cada internamiento se judicializara, de tal modo que, atendiendo al principio de no-maleficencia, sólo en casos excepcionales, formalmente voluntarios pero en los que las capacidades mentales del paciente estuvieran afectadas de manera grave y previsiblemente persistente de no mediar tratamiento, debería considerarse el internamiento como legalmente no consentido.

Aún puede darse un segundo supuesto para el caso de que estas condiciones mínimas de comprensión se den, pero el paciente no acepte un ingreso voluntario, siendo este necesario. A estos efectos deberá aplicarse el principio de beneficencia que exige su internamiento psiquiátrico forzoso procurando el bien del paciente, quedando siempre supeditado a un control judicial según el artículo 763 de la Ley de Enjuiciamiento Civil como garantía del pleno respeto a sus derechos [19].

\section{CONCLUSIONES:}

El análisis precedente nos permite concluir que los principios éticos que mejor se ajustan al objetivo de excelencia en la especificidad de la práctica pericial psiquiátrica son:

- Principio de autonomía: Basado en la libre y consciente elección del informado, siempre que sea competente para ello, respecto a ser sometido a evaluación y a que los datos por él proporcionados sean utilizados en el dictamen de su estado mental en relación con los hechos contemplados en las diligencias judiciales.

- Principio de confidencialidad: Por el cual el perito psiquiatra debe garantizar que los datos obtenidos que no incidan de manera directa en la evaluación del estado mental del informado serán reservados.

- Principio de objetividad: El psiquiatra forense mantendrá una distancia afectiva suficiente para evaluar los diferentes datos de interés con imparcialidad y objetividad ajenas a cualquier condicionante.

- Principio de honestidad: Resulta complementario al anterior, y por él, el perito psiquiatra adquiere el compromiso ético de mantener una independencia científica respecto a quien propone la prueba.

- Principio de verdad y justicia: Entendiendo que el fin último de la actuación médica pericial debe ser el de aportar elementos veraces que permitan a jueces y tribunales impartir justicia.

\section{BIBLIOGRAFÍA:}

I. Mata, P. Tratado de Medicina y Cirugía Legal (I). Madrid: Imp. De Baylli-Bailliere; I.866. p. 208.

2. American Academy of Psychiatry and the Law: Ethical Guidelines for the Practice of Forensic Psychiatry. Adopted May 1987. Revised October 1989, 1991, 1995.

3. W.T. Reich (ed.), Encyclopedia of Bioethics. New York: The Free Press; 1978.

4. Beauchamp TL, Childress JF. Principles of Biomedical Ethics. New York: Oxford Univ. Press, 1979.

5. Gracia, D. Fundamentos de Bioética. pp.505-6. Madrid: Eudema; 1989.
6. LEY 4I/2002, de I4 de noviembre, Básica reguladora de la autonomía del paciente y de derechos y obligaciones en materia de información y documentación clínica. Capítulo I Principios generales. Artículo 2 Principios básicos.

7. Código de Ética y Deontología Médica (1.999).

8. LEY 4I/2002, de I4 de noviembre, Básica reguladora de la autonomía del paciente y de derechos y obligaciones en materia de información y documentación clínica. Capítulo IV El respeto de la Autonomía del paciente. Artículo 8 Consentimiento informado.

9. Marco Ribé, J. y Martí Tusquets, J.L. El peritaje médico-legal psiquiátrico. En Psiquiatría Forense. Barcelona: Espaxs; 2.002. pp. 15-27. 
10. Villanueva E y Gisbert J.A. La peritación médico-legal: introducción jurídica. El método médico-legal. En: Villanueva Cañadas E, editor. Medicina Legal y Toxicología. $6^{a}$ ed. Barcelona: Masson; 2.004. p. $145-55$.

II. Brouardel, P. Cours de Médicine Légale. Paris: J.B. Baillière; 1896-1909.

12. Ley de Enjuiciamiento Criminal (RD de 14 de Septiembre de 1882). Título V "De la comprobación del delito y averiguación del delincuente". Capítulo VII "Del informe pericial". Artículo 478.

13. Gutheil, TG. Ética y Psiquiatría Forense. En: Bloch, S., Chodoff, P., y Green, S.A. La ética en Psiquiatría. Madrid: Triacastela; I.999. pp. 323-39.

14. Bonnet, EFP. De los peritos. En Psicopatología y Psiquiatría Forenses. Buenos Aires: López Libreros; I.983. pp. 174-6.
15. Gutheil, TG. The Psychiatrist as expert witness. Washington DC: American Psychiatric Press; 1998.

16. Jiménez Cubero, F. La Psiquiatría Forense y la ley penal. En: Cuadernos de derecho penal. Madrid: Consejo General del Poder Judicial; I.993 pp. 347-60.

17. Constitución Española de I.978 "Título I, Capítulo II, Sección Ia, artículo 17.I.

18. Conejo Galindo, J. Competencia para consentir la hospitalización voluntaria. INTERPSIQUIS. 2005; [citado 23 de enero de 2.006]. Disponible en: URL: http://www.psiquiatria.com/articulos/psiquiatria legal/21249/

19. Ley I/2000, de 7 de enero, de Enjuiciamiento Civil. 